\title{
posterpresentations in clinicalgenetics
}

\section{2}

Cole-Carpenter Syndrome - A case report. D.J. Amor, A. Bankier. Victorian Clinical Genetics Service, Royal Children's Hospital, Melbourne, Australia

Cole-Carpenter Syndrome is a rare variant of osteogenesis imperfecta characterised by features of both osteogenesis imperfecta and craniosynostosis. Since the initial two cases reported in 1987, there have been only a handful of reports of the condition, none of which have displayed features identical to the initial cases.

We report a case of Cole-Carpenter syndrome with physical and radiological features remarkably similar to the original two cases. The female infant was noted at delivery to have short limbs, brachycephaly and unusual dysmorphic features. A skeletal survey showed generalised osteopenia with shortened and bowed long bones and moderate metaphyseal irregularity. The ribs were short and flared, with a single fracture noted. Skull $X$-ray showed multiple wormian bones.

At follow-up the infant exhibits startling growth failure and progression of dysmorphic features with marked turricephaly. She has significant gross motor delay but normal fine motor and social development. CT scan shows craniosynostosis involving the sagittal, lambdoid, coronal and metopic sutures. Pathological fractures of the bones on either side of the fused sagittal suture are allowing the skull to grow vertically, accounting for the turricephalic appearance. By the age of 14 months the child has experienced two long bone fractures, both with minor trauma.

We present the physical and radiological features of this case and review other reported cases of Cole-Carpenter syndrome.
33

Prenatal diagnosis of dup $(3 q)$ syndrome $\underline{\text { S. K. Barrett }}{ }^{1,2}$, G. S. Kupchik $^{1,2}$, D. Rosa, $a^{1,2}$ S. Haberman ${ }^{1,2}$, T. Burgess ${ }^{1,2}$ M.J Macera ${ }^{2,3}$ and R.S. Verma ${ }^{2,3}$ 'Maimonides Medical Center, Brooklyn, NY; ${ }^{2}$ SUNY Health Science Center at Brooklyn, NY; and ${ }^{3}$ Institute of Molecular Biology and Genetics at InterScience, Brooklyn, NY

There are numerous reports on the duplication of the long arm of chromosome 3, ranging from $3 q 12$ to $3 q$ ter. Atthough the duplicated segments vary in size, a defined clinical phenotype, dup( $3 q)$ syndrome, has been investigated by us and others. A 22 year old G4P0030 (ISPAB, 2ELAB) Caucasian was referred at 31 weeks gestation for fetal anomalies on sonogram including hydrocephalus, bilateral polydactyly, and a possible $\mathrm{CHD}$. Amniocentesis was performed and showed an unbalanced chromosomal rearrangement: 46,XX, $\operatorname{der}(9) \mathrm{t}(3 ; 9)$ (q26.1;p24).ish. 46,XX, der(9)t(3;9)(q26.1;p24) (WCP3+WCP9+), and a positive acetylcholinesterase. Parental chromosomes showed that the father was a carrier of a balanced rearrangement: $46, \mathrm{XY}, \mathrm{t}(3 ; 9)$ (q26.1;24). The baby was born at 42 weeks gestation following prostaglandin induction due to failure to progress. She weighed 4264 grams and cried spontaneously with APGAR scores of $8 / 8$ at 1 and 5 minutes. The major dysmorphic features included: up-slanting palpebral fissures, synophyrs, prominent alveolar ridge, hypertelorism, downturned corners of the mouth, short neck with redundant skin folds, bilateral postaxial polydactyly, a deep sacral dimple and an anteriorly displaced anus with low rectal stenosis. During the surgical correction of the rectal stenosis, a pre-sacral teratoma was excised. The telomeric DNA segment (9p24-pter) lost due to the translocation is generally associated with chromosomal capping rather than phenotypic expression. The clinical manifestations consistent with dup (3q) syndrome are being presented. To our knowledge, teratoma has not been described before as part of the dup $3 q$ phenotype.

\section{4}

Unique Ichthyosis/MR Syndrome with Seizures, Severe Developmental Delay, Failure to Thrive, Mild Dysmorphology and Eye Findings. P.J.Benke', M Lallouz, B. Franco ${ }^{2}$, J. Hannanian', M. Munoz', B. Lam '. 'Univ. Miami, FL and ${ }^{2}$ Telethon Inst. Genetics, Milan, Italy.

We describe three children born to non-consanguinous Puerto Rican parents with severe delay, ichthyosis, minor anomalies, seizures $(2 / 3)$, reactive air-way disease (2/3) severe failure to thrive and unique eye findings. The oldest child, with aimost no skills, is the most affected, but hypoxemia in the neonatal period may have affected his outcome. The two younger siblings have developmental delay that was almost as severe; they could not sit or stand unsupported, crawl or talk. But they are more interactive than their older sibling. All children have mild mongoloid eye slant, epicanthic folds, deep orbits, esotropia, nystagmus, low set ears, long philtrum, small chin and severe failure to thrive. Ichthyosis was confirmed by typical pathological findings. All children have unique eye findings, consisting of decreased visual acuity, esotropia, and horizonal, pendular nystagmus. The pupils dilated paradoxically to light. An electroretinogram in the oldest boy showed an unusual cone response, with a minimal a-wave and prolonged b-wave. An MRI showed an arachnoid cyst and partial ACC. Chromosome, phytanic acid, fatty alcohol dehydrogenase, and long chain fatty acid oxidation testing were negative. Hair analysis was normal, suggesting the children did not have trichothiodystrophy. To determine whether the children had a contiguous gene syndrome consisting of loss of the steroid sulfatase gene and adjacent genetic material, the steroid sulfatase(STS) gene was expanded by PCR utilizing primers at both ends of the gene, and the products isolated and sequenced. This testing was normal. These children appear to have a unique genetic/metabolic disorder which can be distinguished from other Ichthyosis/MR syndromes such as Sjogren-Larson, Tay, Refsum's, Rud's and the Netherton syndromes.

\section{5}

Alpha thalassemia major: counseling for the possibility of long-term survival J.Bojanowski. T.Singer, L.Styles, E.Vichinsky, D.Foote, K.Quirolo. Children's Hospital Oakland, CA.

Deletion of all four alpha globin genes most often leads to fetal demise or stillbirth secondary to hydrops fetalis. In recent years, long-term post-natal survival bas been reported in a handful of cases, some following stem cell transplantation and/or intrauterine transfusion. Given the possibility of treatments that may enable long-term survival and ameliorate some associated sequelae, there are several issues to consider when counseling the couple with an affected fetus. We report a Laotian female longterm survivor with alpha thalassemia major, a case that illustrates several issues.

Our patient was born to a 23 year-old G2,P1-2 Khmu-speaking woman whose carrier status was not previously identified. Fetal ultrasound at $373 / 7$ weeks revealed intrauterine growth retardation. hepatomegaly, abdominal ascites, and an enlarged umbilical vein. Ultrasound at $382 / 7$ weeks additionally noted fetal cardiomegaly. The infant was delivered by emergency cesarean section at that time secondary to hydrops and fetal distress. Birth weight was 2200 grams, length was $43 \mathrm{~cm}$, and head circumference was $31 \mathrm{~cm}$ (all < 3rd percentile). Immediate intubation and ventilator support were required, as well as a packed red cell transfusion and an exchange transfusion. DNA analysis revealed homozygosity for the Southeast Asian two alpha globin gene deletion (- SEA / - SFAA ). The child, now 2 years of age, has since been on chronic transfusion therapy to maintain a $\mathrm{Hb} \mathrm{H}$ level of $20-35 \%$. Formal testing has shown normal development and appropriate growth parameters. At age 8 months, an echocardiogram identified a $9-10 \mathrm{~mm}$ atrial septal defect, which awaits surgical repair. Bone marrow transplantation is an option for this child who has an HLA-matched sibling. The role of family members has been tremendously important to the process of informed medical decision-making and in the provision of appropriate care.

This case illustrates several important counseling issues. Appropriate carrier screening is necessary prior to conception or early in pregnancy. Once an affected fetus is identified, feasibility of in-utero treatment options should be reviewed along with a discussion of possible maternal risks and future clinical implications for the affected child. Postnatal transfusion requirements are similar to those for a child with beta thalassemia major. Iron overload and chelation therapy should be discussed, as well as the possibility of bone marrow transplantation given a matched donor. The increased risk for developmental delay and congenital malformations in alpha thalassemia major should also be considered. In all cases, parents should be involved in the decisionmaking process, and cultural and social considerations should be addressed. 